\title{
Alcances y límites del análisis de redes sociales en la actualidad: una reflexión sobre tres deseables extensiones
}

\author{
Jorge E. Miceli
}

Recibido: 11 de noviembre de 2010 Revisado: 16 de diciembre de 2010 Aprobado: 18 de enero de 2010

\section{ReSUMEN}

El análisis de redes sociales (ARS) ha experimentado un crecimiento incesante en los últimos años, generando expectativas de consolidación que exceden nuestra capacidad reflexiva. En este artículo he abordado dos tipos de limitaciones devenidas de distintas etapas de cualquier proceso investigativo. Por un lado hago referencia a perspectivas epistemológicas que implican ciertas limitaciones al momento de pensar la investigación sobre redes sociales. Por el otro, tengo en cuenta algunas imposibilidades surgidas del desarrollo práctico del software y de los dispositivos y heurísticas de cálculo usados. En particular, destaco aquí las cuestiones relacionadas con la ausencia de una perspectiva temporal en ARS, con el uso de la intuición naturalista en la representación de las redes y con la suposición de la aleatoriedad relacional como la norma típica en las redes que de forma empírica existen.

\section{Palabras clave}

Análisis de redes sociales, aleatoriedad relacional, temporalidad, intuición naturalista. 


\title{
Scope and Limits of Social Network Analysis Today: a Reflection on Three Desirable Extensions
}

\author{
Jorge E. Miceli
}

\begin{abstract}
Social Network Analysis (SNA) has experienced constant growth in recent years, raising expectations of consolidation that exceed our capacity for reflection. I consider, in this article, two kinds of limitations which occur in different stages of any research process. On the one hand, I refer to epistemological perspectives that involve certain limitations when thinking about social networks research. On the other hand, I consider some impossibilities arising from the practical development of software and used computing devices and heuristics. In particular, I address here the issues related to the lack of time perspective in ARS, the use of naturalistic intuition in the representation of the networks and the assumption of typical randomness as the relational standard empirically existing networks.
\end{abstract}

\section{KEYWORDS}

Social network analysis, relational randomness, temporality, naturalistic intuition. 
Una dificultad inminente para realizar muchos de nuestros trabajos e investigaciones es lo que se denomina como aquella desviación o limitación del pensamiento científico que tiende a reducir relaciones $u$ objetos nuevos a los ya conocidos. Debemos reconocer, y lamentar, que este tipo de obstáculo tiene una larga presencia y un profundo peso en nuestra historia laboral.

Gastón Bachelard

\section{INTRODUCCIÓN}

Lejos de conformarse como una perspectiva estática, y acompañado de un desarrollo impresionante del software asociado a su implementación, el análisis de redes sociales (de aquí en adelante ARS) ha experimentado un crecimiento incesante en los últimos años, generando expectativas de consolidación que exceden largamente a la capacidad reflexiva de sus practicantes. En otro momento he señalado algunos inconvenientes relacionados con la tendencia global a reificar esta perspectiva y otorgarle cierta infalibilidad a su desempeño (Miceli, 2008), pero me interesa, en este espacio, destacar algunas especificidades que hacen a los límites del ARS en su ejercicio profesional reciente.

La constatación de estos límites no es, ni remotamente, un proceso enteramente inventivo de mi parte. Algunas veces me he basado en comentarios externos y otras en enfatizaciones y precisiones de mi autoría, pero lo que me interesa es poner estas cuestiones en el centro de la escena y que motiven una reflexión de los profesionales del ARS.

Entiendo, en este sentido, que hay dos tipos de limitaciones devenidas de distintas etapas de cualquier proceso investigativo. Por un lado hay perspectivas epistemológicas que implican ciertas limitaciones al momento de pensar investigaciones de redes sociales. Estas posiciones conciernen al momento del planeamiento de una investigación, pero se proyectan fuertemente al resto de las fases de este proceso. En segunda instancia existen imposibilidades surgidas del desarrollo práctico del software y de los dispositivos y heurísticas de cálculo usadas, lo que ha sesgado el curso de las investigaciones hacia territorios muy transitados de los cuales cuesta cada vez más apartarse. El efecto general de todas estas constricciones es que cada vez más el ARS aparece como una herramienta cuya capacidad de producir enfoques novedosos, atravesado ya de cierto deslumbramiento inicial, aparece considerablemente mitigada.

En este espacio me ocuparé de algunas limitaciones epistemológicas que asigno como obstáculos centrales en la evolución del ARS. Estas limitaciones no son excluyentes de otras que tienen una dimensión técnica más directa y casi urgente, como por citar algunos ejemplos, el subdesarrollo del análisis de redes de más de un modo, de las redes de relaciones negativas o el soslayo de la dimensión atributiva en el análisis pero, por limitaciones contextuales, no las tocaré aquí. Por otro lado, cabe aclarar que el carácter epistemológico de estas constricciones no le quita su traducción fuertemente técnica en el plano del software disponible: las extensiones que planteo cuentan casi sin excepción, con un soporte inexistente, bastante pobre o poco integrado en el plano de los programas informáticos que facilitarían su difusión.

Sin embargo, no pretendo que esta semblanza implique reflexiones meramente 
críticas. A la par que intento destacar lo que todavía no se ha hecho, el concepto de una posible "extensión" conlleva la potencialidad evidente de un rumbo a seguir.

\section{LA EXTENSIÓN}

\section{HACIA LO NO ALEATORIO: \\ EL CUESTIONAMIENTO}

\section{DEL AZAR RELACIONAL COMO}

\section{LA CONDICIÓN PREDOMINANTE} DE LAS REDES REALES

Sabemos que hasta hace poco tiempo había muy poca investigación centrada en las diferentes topologías de redes existentes, y dentro del ARS clásico era posible calcular la densidad, contar sus componentes y medir su diámetro, pero no demasiado más que esto. En lo que tiene que ver con la forma general de las redes empíricas se suponía que la abrumadora mayoría de ellas deberían mostrar enlaces al azar. Esta visión apriorística, sustentada en parte en la intuición matemática y en el valor de uso de los modelos disponibles, tornaba factible aplicar los algoritmos específicamente diseñados para grafos enlazados al azar por el matemático húngaro Paul Erdös hace unos 40 años. Como he dicho en otro momento:
En 1998, Laszlo Barabasi, Jeong y Albert, trabajando en la realización de un mapa de la Web, albergaban la razonable esperanza de que el grafo obtenido en sus análisis fuera similar a una red con enlaces distribuidos al azar. Ello involucraba una distribución de frecuencia de enlaces de los nodos en los que coincidiera aproximadamente la media, la mediana y el modo, con forma similar a una curva normal o de Gauss, es decir, con muchos nodos con una cantidad de enlaces cercanos a la media y bastantes menos páginas en ambos extremos de las frecuencias distribución de enlaces. Para su sorpresa encontraron que la gran mayoría de las páginas de la Web tenía menos de 4 enlaces mientras un $0,01 \%$, tenía más de 1.000 , pero también advirtieron que este desequilibrio o inequidad distributiva parecía ser esencial para sostener la conectividad total que exhibía Internet. La distribución de páginas con cierta cantidad de enlaces seguía lo conocido como "Ley de potencia", haciendo que la probabilidad de que algún nodo esté conectado con $\mathrm{k}$ otro nodo sea proporcional a $1 / \mathrm{k}$ elevado a la $\mathrm{n}$. Tal distribución no tiene un pico como la curva normal, sino que es una función continuamente decreciente y si se dibuja en un gráfico con una escala doble logarítmica aparece como una línea recta (Barabasi, et ál., 2003) (Miceli, 2007, p. 8). 
Figura 1. Representación estándar de redes aleatorias e independientes de escala

A) Aleatoria

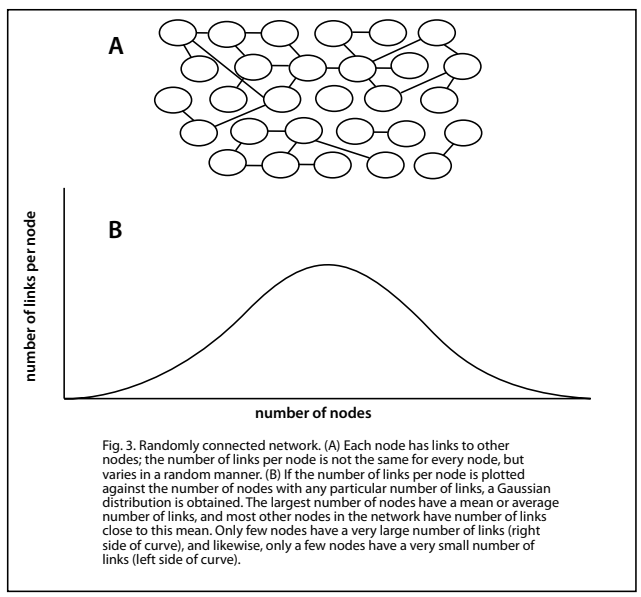

B) Independiente de escala

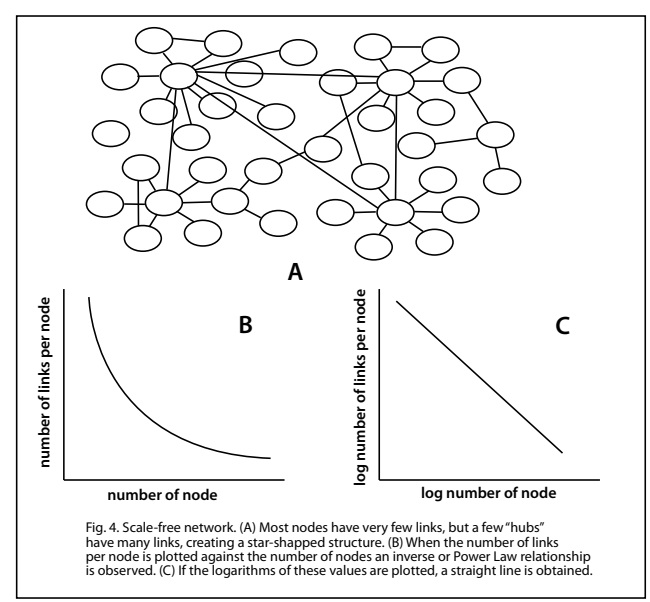

Fuente: Smith Andrew P. en Small Word, Big Cosmos, recuperado desde [http://207.44.196.94/ wilber/smith17.html].

Este tipo de red se conoce como "Independiente de Escala" (Scale-Free Network) ya que no tiene un valor típico bien definido (una escala que la caracterice) dentro del cual su morfología sea reconocible. Después del trabajo pionero de Barabasi se empeza- ron a descubrir redes libres de escala en muchos dominios (enlaces entre aeropuertos, cableado eléctrico, relaciones sexuales entre personas, coautorías de artículos). Barabasi y Albert intentaron explicar esta ubicuidad de las redes en el mundo real, y lo hicieron apelando a dos mecanismos: crecimiento y enlace preferencial. Su formulación sostiene que las redes independientes de escala van creciendo con el tiempo y los nuevos nodos que se adosan se enlazan preferentemente con los ya existentes en función de su número previo de conexiones. Estos mecanismos bastan para explicar el surgimiento de las redes independientes de escala, su mantenimiento en diferentes tamaños (escalas de la red) y también su presencia muy frecuente.

El caso es que los descubrimientos de Barabasi y Albert afectaron seriamente lo que se pensaba sobre las redes reales, al punto que de a poco su estudio empezó a demostrar que la aleatoriedad es un muy mal modelo de ellas. Por otro lado, el concepto de mundo pequeño aplicado a las redes ha recibido un impulso decisivo de la mano de Duncan Watts, quien estudiando la topología de estructuras reticulares reales, ha logrado desentrañar el modo en que, en los casos estudiados, aquellas que tienen estas características cuentan con una navegabilidad elevada y una resistencia a los ataques mucho mayor que las que muestran con conformación aleatoria (Watts, 2004).

Entonces, las investigaciones de Watts tuvieron un doble correlato teórico y empírico; a nivel teórico implican que el "recableo" de una red convencional, de trazado regular, puede producir rápidamente, como muestra la figura 2, una estructura de "pequeño mundo" con sólo algunos caminos agregados. 
Figura 2. Grilla Regular y Grilla Regular "recableada" de Strogatz-Watts
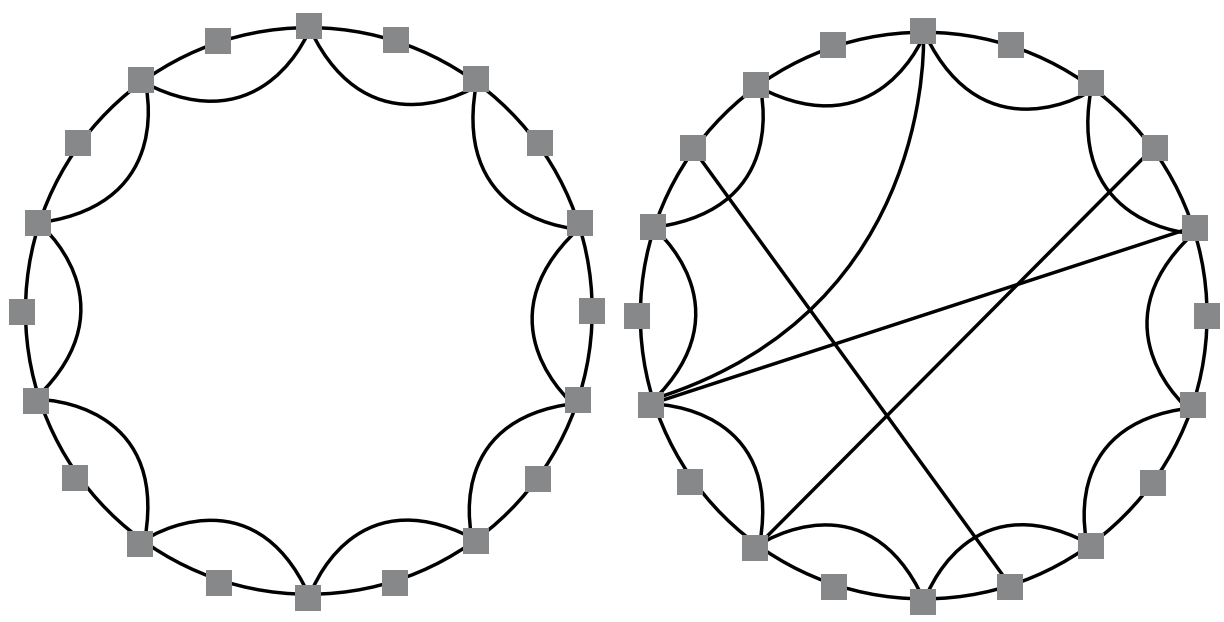

Fuente: Reynoso, 2008.

A nivel empírico estos hallazgos implicaron la constatación de que las redes más resistentes son aquellas que están "clusterizadas", es decir, aquellas en las cuales la distribución de conexiones es fundamentalmente desigual. Como sostiene Carlos Reynoso:

La primera lección a sacar aquí, epistemológicamente hablando, es que el azar es un pobre modelo de las estructuras de red que se encuentran en la vida real; más todavía, lejos de constituir una heurística útil, el azar es en estos escenarios un modelo inhibidor que impide abordar y comprender estructuraciones esenciales de la realidad.

Las estructuras de los pequeños mundos son todo lo contrario al ruido blanco; son además configuraciones tan robustas como delicadas, invisibles al muestreo y a la percepción sincrónica. Dado que una sensible mayoría de los métodos estadísticos en diversas disciplinas presuponen que la muestra de base está regida por el azar y es representativa del conjunto, corresponde tal vez repensar o tomar con extremada prudencia dichos métodos cuando de redes y complejidad se trata (Reynoso, 2008, p. 132).

Es sorprendente constatar, en definitiva, que el aluvión de las redes libres de escala o de pequeño mundo se ha extendido a diferentes campos, cuestionando de manera absoluta el predominio de la aleatoriedad incluso en el plano de la química o la física de fluidos.

No me interesa en este espacio constatar la genealogía de estas ideas, que remontan, por lejos, al legendario experimento de Stanley Milgram de 1967 acerca de los seis grados de separación, y que se pierden en una maraña de referencias literarias bastante anteriores. Tampoco me interesa postular la prevalencia apriorística de estas redes o de las aleatorias o regulares como generadoras de hipótesis nulas. Lo que señalo, sin embargo, es la necesidad de comenzar a colocar al análisis de las distribuciones empíricas de las redes como uno de los aspectos 
centrales de su estudio, ya que implican supuestos estadísticos y generativos de distinto tipo. En definitiva, diferentes configuraciones actuales de las redes, la mayoría de ellas alejadas del simple azar, pueden remitir a diferentes historias de su evolución, y eso ha sido soslayado en una medida no tolerable en los abordajes convencionales de ARS.

\section{LA EXTENSIÓN HACIA LO NO INTUITIVO: LA NECESIDAD DE CONSTRUIR REDES QUE SUPEREN EL MAPEO NATURALÍSTICO DE NODOS Y LAZOS}

En la mayoría de las implementaciones científicas y de consultoría, las redes mapeadas se corresponden con propiedades autoevidentes de nodos y lazos, cuando al menos en su expresión potencial cualquier cosa puede cumplir estos roles. A pesar de que el ARS suministra una plataforma refinada para analizar los datos reticulares, los problemas de diseño de estas redes y su trivialidad teórica no son problemas atribuibles al método, sino a la transposición naturalística que se hace de la información real.

En efecto, por ejemplo, si analizamos relaciones entre personas, parecería que no hay que dedicarse un segundo a pensar en profundidad los supuestos de isomorfismo en la representación de esta red, ya que aquellas entidades que se nos "imponen" perceptivamente (las personas y sus lazos) van a ser objeto de representación directa. De esta forma, todas las maneras en que creativamente se puedan concebir estas estructuras reticulares, considerando como nodos a algo distinto a las personas y como relaciones a algo distinto a las conexiones de intercambio o parentales, por ejemplo, son pasadas por alto sin meditar sobre las consecuencias globales de estas exclusiones.

Desde que los motivos de esta operatoria no son nada triviales cognitivamente, y deben tener que ver con la eficacia de los grafos como dispositivos didácticos de alto impacto visual. Sin embargo, nos encontramos aquí con una limitación seria al momento de improvisar nuevas formas de ver las cosas, y es la fuerte carga empirista del ARS como dispositivo de análisis.

El rótulo "redes sociales" implica, incluso, una seria implicación realista. En rigor de verdad, la teoría de grafos reconoce aplicaciones que exceden largamente a objetos de estudio a realidades sociales, pero la noción usual de "red social" conlleva, en su interpretación técnica, supuestos que a poco de examinados resultan erróneos: en efecto, los nodos pueden ser cualquier cosa, no sólo grupos de personas, clubes o países, sino lo que concebimos como relaciones o incluso aquello que normalmente calificamos como atributos de una estructura.

Como verificamos en esta cita de Wikipedia, toda la terminología de análisis de redes tiene un sesgo naturalístico que afecta notablemente el tipo de conclusiones a extraer:

Los analistas estudian la influencia del todo en las partes y viceversa, el efecto producido por la acción selectiva de los individuos en la red; desde la estructura hasta la relación y el individuo, desde el comportamiento hasta la actitud. Como se ha dicho, estos análisis se realizan bien en redes completas, donde los lazos son las relaciones específicas en una población definida, o bien en redes personales (también conocidas como redes egocéntricas, aunque no son exactamente equiparables), donde se estudian "comunidades 
personales". La distinción entre redes totales/completas y redes personales/ egocéntricas depende mucho más de la capacidad del analista para recopilar los datos y la información. Es decir, para grupos tales como empresas, escuelas o sociedades con membrecía, el analista espera tener información completa sobre quien está en la red, siendo todos los participantes egos y alteri potenciales. Los estudios personales/ egocéntricos son conducidos generalmente cuando las identidades o egos se conocen, pero no sus alteri. Estos estudios permiten a los egos aportar información sobre la identidad de sus alteri y no hay la expectativa de que los distintos egos o conjuntos de alteri estén vinculados con cada uno de los otros (Wikipedia, recuperado el 28 de julio de 2010 desde [http://es.wikipedia.org/ wiki/Red_social]).

En términos más estrictos, este tipo de comentarios invoca ideas muy sesgadas respecto a qué elementos pueden ser considerados nodos o vértices y cuáles lazos o vínculos. En un repaso muy breve tenemos "acción selectiva de los individuos en la red", "lazos en una población definida", "redes personales (...) como sinónimo de redes egocéntricas, etc." Todas estas afirmaciones implican una visión fuertemente lineal de la aplicación del ARS, traduciendo a un orden matricial relaciones que parecen evidentes en el plano empírico. El inconveniente más importante derivado de esta concepción es la desproblematización conceptual en la construcción primaria de la red, ontologizando componentes que merecerían una interpretación no naturalística. En este caso, la acción selectiva podría estar referenciada en grupos y no en individuos, o en díadas o tríadas de ellos, etc. Por otro lado, lo que se denomina "población" podría ser un conjunto de eventos, de pertenencias de clase, de filiaciones territoria- les, etc. En tercer lugar, el concepto de red egocéntrica no remite formalmente a redes personales, sino a los lazos que se vinculan a un único nodo específico por vez y más allá de lo que sean estos nodos. Desanclar la definición de lo que es una red, de estas afirmaciones de sentido común es acaso el mayor desafío pendiente, pero los beneficios pueden ser amplios.

Como ejemplo de las posibilidades que genera un diseño no naturalístico, cito un caso abordado por matemáticos para solucionar un problema concreto:

Dada una colección de circuitos [tours]
de camiones de recolección de basura
¿es posible asignar cada circuito a un
día de la semana (que no sea domin-
go), tal que si dos circuitos visitan un
sitio en común lo hagan en diferentes
días? [...].

Para formular este problema en términos de teoría de grafos, si $G$ es el grafo de circuito (el grafo cuyos vértices son los circuitos) y si existe una arista entre dos circuitos sí y sólo si ellos visitan un sitio en común, el problema es equivalente al que sigue: ¿es posible asignar a cada vértice (circuito) uno de los seis colores (días), de modo tal que si dos circuitos se unen con una arista (visitan un sitio en común) obtienen colores diferentes? Esta pregunta deviene entonces: ¿es el grafo en cuestión 6-colorizable? (Roberts 1978, p. 49 \& Reynoso 2009).

Para intentar esquematizar este problema, el obstáculo más inmediato que enfrentamos aquí es concebir el grafo que representa a este diseño de modo intuitivo, lo que se nos ocurre es asignarles a las calles el papel de lazos, y a los encuentros entre ellas el de nodos. A lo sumo, como señala Reynoso, se pueden invertir estas asignaciones, pero la representación sigue siendo naturalística aunque cambien los roles. 
Tabla 1. Tipos de representación asociados al problema de la recolección de basura

\begin{tabular}{|l|l|l|}
\hline $\begin{array}{l}\text { Representación naturalística } \\
\text { directa o de primer orden }\end{array}$ & $\begin{array}{l}\text { Representación naturalística } \\
\text { indirecta o de segundo orden }\end{array}$ & Representación no naturalísitca \\
\hline Nodos: esquinas & Nodos: calles & $\begin{array}{l}\text { Nodos: circuitos que enlazan } \\
\text { sitios }\end{array}$ \\
\hline Lazos: calles & Lazos: esquinas & Lazos: sitios visitados en común \\
\hline
\end{tabular}

El algoritmo por el cual se resuelve este problema, recurriendo a la teoría de grafos y no al ARS, subvierte y cuestiona esta lógica, anclada en la representación usual del lenguaje. En el escenario de esta solución, los nodos se convierten en circuitos, y las aristas o lazos se producen cuando dos circuitos visitan un sitio en común. Los días de la semana, indicadores de una representación diacrónica del tiempo, se instancian en el modelado de red como seis nodos diferentes que coinciden con cada uno de los circuitos (menos el domingo).

Como observamos en la tabla 1, la generación de una representación no naturalística de la red no tropieza con obstáculos cognitivos menores, ya que la definición de nodos y lazos implica pensar en conceptos que vinculan de manera antiintuitiva objetos de la vida cotidiana. Un nodo es aquí un objeto formal compuesto por sitios enlazados a través de trayectos autónomos, y el lazo se produce cuando dos circuitos se intersectan. En la figura 3, en definitiva, cómo la representación del problema puede virar desde el gráfico de la izquierda, que constituye una transición formal derivada de una primera imagen naturalista, hacia una esquematización completamente alejada de lo que se intuye sobre una red, que es la mostrada en el dibujo de la derecha. En esta última los caminos que se visualizan, y que permiten resolver el problema del recorrido común, pensando en términos mucho más eficaces, son en realidad sitios en común que dos circuitos cualesquiera tienen entre sí.

Una de las dificultades más importantes aquí es romper con las constricciones lingüísticas y mentales que hacen que pensemos a los nodos como puntos individuales y a los lazos como sucesiones de puntos, porque eso nos transforma en esclavos de una geometría proyectiva que impone límites a la flexibilidad de la representación.

La coloración de grafos, que es la disciplina que se encarga de resolver estas cuestiones, ha evolucionado independientemente y conforma acaso un episodio más de la divergencia actual que Reynoso señala entre teoría de grafos y teoría de redes sociales, que, luego de aquel origen común sustentado en la invención euleriana, han tomado caminos bastante disímiles. 
Figura 3. Diagrama de caminos y expresión simplificada
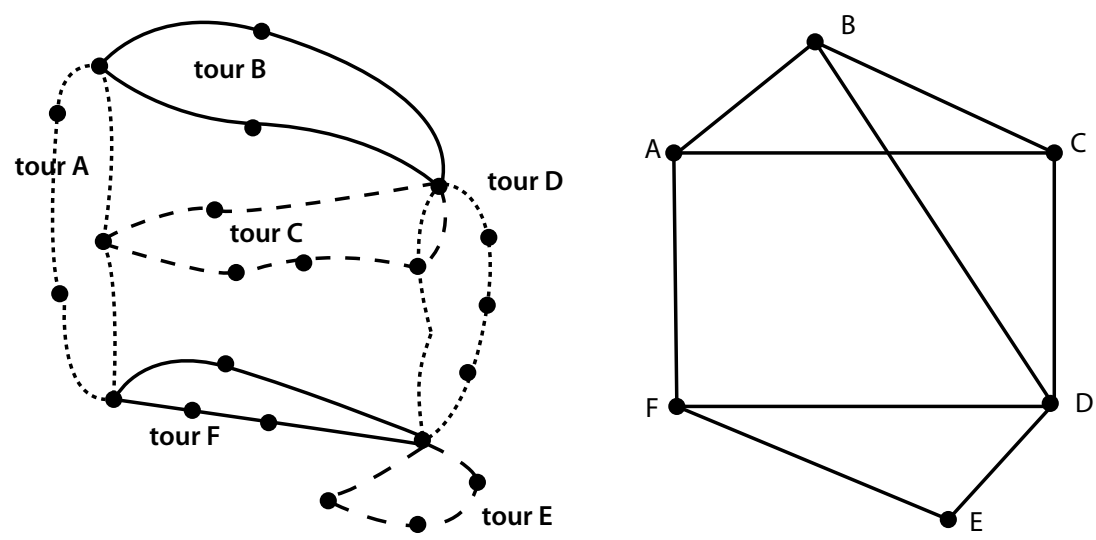

Fuente: Reynoso, 2008.

Estimo que un gran progreso sería, para el análisis de redes, incorporar modalidades de representación no naturalistas, pero creo que es necesario hacer dos señalamientos respecto a la productividad relativa de este esfuerzo:

- Los planteos de coloración de grafos responden, sin dudas, a estrategias resolutivas que operan clasificando los modos de recorrer una estructura, pero habría que especializar estos algoritmos para procedimientos de análisis y diagnóstico que deben ser replanteados, a estos efectos, como tentativas de solución, por ejemplo, de un problema taxonómico o categorial más complejo. Esta operatoria es accesible en términos algorítmicos teóricos, pero muy poco accesible en términos concretos en tanto no se genere software que la incorpore al stock de procedimientos de análisis. Un inconveniente nada desdeñable para este objetivo es que los analistas de redes no sólo plantean sus problemas de modo naturalista, sino que usan programas confeccionados desde el ARS y no desde la teoría de grafos. Me pregunto, en este sentido, la trasposición naturalista ha impregnado no sólo la concepción conceptual de la red, sino sus dispositivos básicos de análisis.

- A pesar de estas observaciones se debe reconocer que se han desarrollado muy buenos estudios con diseños reticulares de orden naturalista, y no debemos dejar de lado para nada ese camino. Todos los estudios clásicos de redes sociales de la Escuela de Manchester, el clásico estudio de Padgett de las familias florentinas (Padgett, 1999), y hasta los hallazgos de Mark Granovetter sobre los lazos débiles han sido plasmados con estas perspectivas, aportando importantes progresos a las ciencias sociales a nivel técnico- 
metodológico e introduciendo novedades conceptuales de alto impacto teórico (Granovetter, 1973). Si nos proponemos incorporar los hallazgos de la teoría de grafos al ARS, tendríamos que contemplar la complementariedad funcional con las perspectivas preexistentes.

\section{LA EXTENSIÓN HACIA LA DIACRONÍA: LA NECESIDAD DE PRIORIZAR LA TEMPORALIDAD EN LOS ANÁLISIS CONVENCIONALES}

La diacronía es otra asignatura pendiente del análisis de redes, pero recién ahora estamos teniendo conciencia de las dimensiones de esta constricción.

Si bien el software SIENA (Simulation Investigation for Empirical Network Análisis), por ejemplo, dispone de interfaces informáticas para mapear redes en el tiempo, sus aplicaciones no se han difundido y hoy en día el ARS sigue siendo un dispositivo de análisis eminentemente sincrónico.

Recién con la introducción de los modelos basados en cadenas de Markov, que superaron a los viejos modelos estadísticos de tipo loglinear, la dinámica temporal ha adquirido el marco de desarrollo acorde a su relevancia intrínseca (De Federico, 2005, pp. 155-156).

En este sentido, cabe recordar que este modelo de análisis y su software asociado, basados en la idea de cadenas markovianas y desarrollado por Tom Snijders (2001-2005) "proponen un modelo estadístico de análisis de datos longitudinales para redes totales" (De Federico, 2005, pp. 155-156).

Si bien el modelo Siena está basado en estos principios, muestra, a mi entender, una se- rie de importantes limitaciones operativas y epistemológicas:

1) Depende de la carga estadística completa de al menos dos momentos de la red, ya que no tiene capacidad generativa autónoma ${ }^{1}$. Esto implica que el software opera con base a datos correspondientes, al menos dos instancias temporales, y trata de inducir, de manera exploratoria, las causas de las variaciones entre estas etapas (De Federico, 2005, pp. 155-156), pero no puede generar libremente y a partir de parámetros específicos, las fases intermedias anteriores o posteriores de la red.

2) Implica presupuestos de evolución altamente cuestionables, entre ellos, la idea de que "los actores tienen un conocimiento total del estado de las relaciones en la red" actores tienen la misma probabilidad de realizar un cambio" (De Federico, 2005, p. 159). Estos presupuestos no se pueden parametrizar, sino que fuerzan condiciones de funcionamiento fijas para todos los procesos.

3) No es una interfaz abierta de programación, una plataforma de desarrollo,

1 Según De Federico: "Se consideran datos longitudinales de redes totales siguiendo una serie temporal $x(t), t, t\{t 1, \ldots, t M\}$ para, en principio, un número constante de actores $\{1, \ldots, n\}$. Los momentos de observación están ordenados: $11<t 2,<\ldots<\mathrm{M}$. El número $\mathrm{M}$ de observaciones es por lo menos 2." (De Federico 2005: 157).

2 En relación a este supuesto, y como señala la misma autora "El postulado sobre el conocimiento total de la estructura de la red por los actores no es en absoluto realista, particularmente para grandes redes. Es muy difícil conocer el estado preciso de las relaciones entre actores de una red, como mucho, es posible representarse su estado, lo que bien puede no coincidir con la realidad. Para las relaciones de carácter privado, por ejemplo las relaciones de amistad, que pueden desligarse del contexto inicial de formación, no siempre es posible conocer la existencia de un lazo particular entre dos personas más allá de su relación cordial, incluso cuando su interacción es parcialmente observable" (De Federico, 2005, p. 161). 
sino que conforma un paquete cerrado de opciones que permiten inferir inductivamente causas de variación a partir de una serie cerrada de alternativas.

4) No ofrece un paquete de análisis integrado al modelo más allá de los parámetros que regulan los procesos de cambio.

Sin dudas que el modelo SIENA es una vía interesante de trabajo, pero al no conformarse como una completa plataforma de desarrollo, y al ser una herramienta de inferencia no generativa, no personalizable y de propósito global (no orientada a casos), sus prestaciones ofrecen importantes límites para el estudio de dinámicas procesales específicas.

Lo que está introduciendo un cambio, en este aspecto, es el enfoque reticular basado en agentes, en el cual las redes se despliegan en el tiempo y comienzan a implicar principios generativos de diferente tipo. Esto habilita la posibilidad de vincular estructuralmente tipos de fenómenos empíricos con procesos generativos por un lado, y tipos de redes por el otro.

Los ejemplos que se ajustan a esta perspectiva son bastantes en el caso del software NetLogo ${ }^{3}$, en la actualidad, en una plataforma de desarrollo capaz de incluir a las redes como uno de sus principios generativos.

Aquí solo expondré, por obvias cuestiones de espacio, las particularidades del modelo Team Assembly, que como señalan sus autores:

...illustrates how the behavior of individuals in assembling small teams

3 Desarrollado por Uri Wilensky para el "Center for Connected Learning and Computer-Based Modeling "(CCL) de la Northwestern University de Evanston, Illinois, EE.UU. for short-term projects can give rise to a variety of large-scale network structures over time. It is an adaptation of the team assembly model presented by Guimera, Uzzi, Spiro \& Amaral (2005). The rules of the model draw upon observations of collaboration networks ranging from Broadway productions to scientific publications in psychology and astronomy. Many of the general features found in the networks of creative enterprises can be captured by the Team Assembly model with two simple parameters: the proportion of newcomers participating in a team and the propensity for past collaborators to work again with one another (Bakshy \& Wilensky, 2007).

Como sabemos, el análisis de redes científicas ha sido un campo floreciente de análisis reticular, dando lugar a la rama conocida como bibliometría (Crane, 1972).

Como sostiene José Luis Molina, los colegios invisibles pueden definirse dentro de,

...una tradición de estudios iniciada por D.J. Solla Price (Little Science, Big Science, 1963, citado en Callon, 1995), según la cual la investigación científica parece seguir una curva en forma de S (curva logística) en la que es posible identificar una etapa inicial de aparición del paradigma, una segunda etapa de multiplicación de las contribuciones y, asociada a la aparición de "colegios invisibles" o círculos de investigadores influenciados por unos pocos investigadores de alta productividad, una tercera fase de madurez y una última de estancamiento.

Estos trabajos se basan principalmente en el recuento de citas, aunque también incluyen, como es el caso del trabajo de Crane, otros tipos de relaciones (dirección de tesis doctorales, comunicación informal e influencia en la selección de problemas). 
Crane asimila "colegio invisible" a círculo social, en el sentido que los integrantes de un círculo social solamente conocen a una parte del total pero están influidos por personas con las que no están conectadas directamente. Por "conectividad" entiende la existencia de un camino que uno a dos científicos en una dirección determinada, independientemente de su longitud. Por tanto, el énfasis de esta aproximación se centra en el establecimiento de la red total en la cual se hayan insertos los autores, sean conscientes o no, y en el cálculo de la conectividad existente (Molina, et ál., 2002, p. 2).

La red de colegios invisibles, como campo de investigación autónomo, tiene en cuenta estas redes de influencias que pueden ser directas e indirectas, y que generan complejas estructuras en su evolución.

Sin embargo, el abordaje de estos vínculos en el tiempo no había sido considerado, hasta ahora, como objeto de estudio autónomo. Siendo el crecimiento de las redes científicas un fenómeno harto conocido por la comunidad académica, las preguntas sobre el proceso generativo que las crea, sobre los factores diferenciales que inciden en su desarrollo, sólo podían ser planteadas hasta ahora de modo indirecto y a través de fotos parciales sometidas a toda la batería de cálculos del ARS.

Las nuevas interfaces de modelización de estos procesos proveen la posibilidad de parametrizar a voluntad la evolución de una red, chequeando los cambios que experimenta ante combinaciones inéditas de estos valores. En este caso, en cada nueva instancia de la red, un nuevo equipo de investigación se conforma. Los miembros de cada equipo pueden ser "novatos" (newcomers) investigadores que no tienen participación previa en ningún equipo, o "titulares" (incumbents), científicos que tienen experiencia previa trabajando en otros equipos. Hay en estas simulaciones dos parámetros centrales que van regulando los eventos del proceso; el primero $(P)$ es la posibilidad de que cada miembro nuevo sea titular; el segundo $(Q)$ determina la probabilidad de que el nuevo miembro, si no es novato, sea elegido del conjunto de colaboradores previos de alguien titular que ya esté en el equipo. De otra forma, el nuevo miembro será elegido aleatoriamente dentro de los titulares de cualquier proyecto. Cuando un proyecto nuevo es creado, todos los miembros están vinculados entre sí. Si un agente no participa en un nuevo equipo durante un período prolongado de tiempo, el agente y sus enlaces se quitan de la red. Los agentes reunidos en un nuevo equipo son de color azul si son recién llegados, y amarillo si son titulares. Los círculos grises más pequeños representan a los que no están colaborando actualmente. Los enlaces indican la experiencia de los miembros en su época más reciente de colaboración. Por ejemplo, los vínculos entre los agentes de color azul indican que dos agentes colaboraron como recién llegados. Los enlaces verde y amarillo corresponden a colaboraciones específicas entre titulares y recién llegados, y entre titulares y titulares, respectivamente. Por último, los enlaces rojos indican que los agentes han colaborado entre sí varias veces.

En cada momento del tiempo, que aquí tratamos como "pasos", un nuevo equipo conformado por cuatro miembros se suma a la red existente. El tamaño de cada nuevo equipo de investigación puede ser regulado de manera parametrizada, modificando el parámetro "team size". 
Figura 4. Tipos de colaboración en redes científicas según modelo de Team Assembly

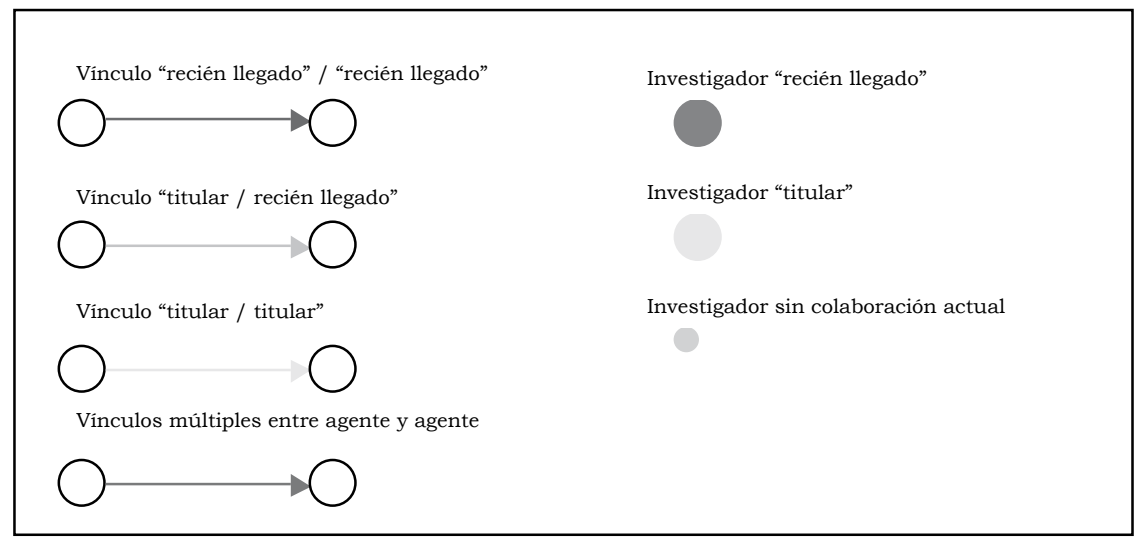

Otro aspecto parametrizable es la variable que se designa como MAX-DOWNTIME, que establece el número de etapas o el tiempo en que el agente permanecerá en la red sin colaboraciones hasta que llegue el momento del retiro. Lo interesante aquí es cómo la gestación de la red puede ser observada en un tiempo simulado que sustituye al tiempo real:

Figura 5. Pasos 1, 2 y 3 en evolución de la red de Team Assembly
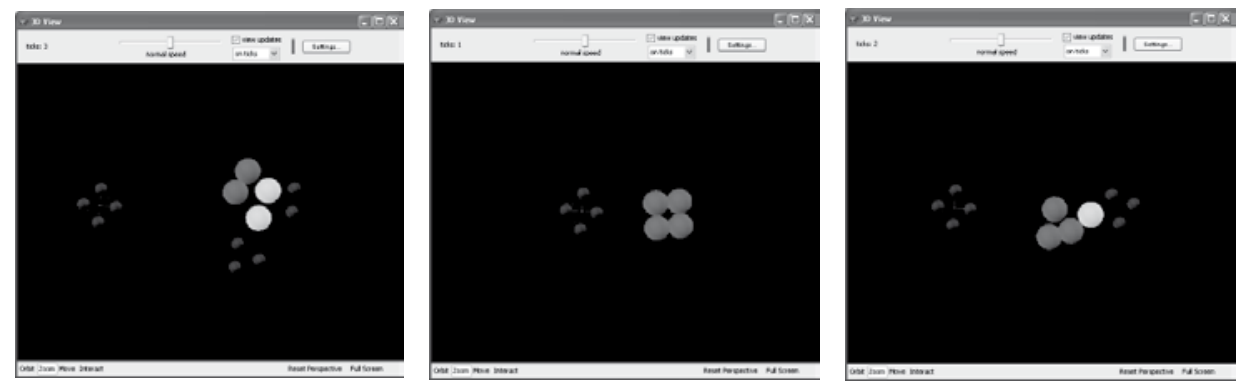

En el primer paso se generan dos conglomerados, uno de investigadores sin colaboración actual coloreados de gris, y otro conglomerado formado por recién llegados coloreados de azul.

En el segundo momento un investigador titular opera de nexo entre el conglomerado de recién llegados y el de investigadores sin colaboración, y el conglomerado de investigadores sin nexos, ya existente, permanece aislado.
En la tercera etapa un investigador recién llegado se transforma en titular, uniendo a recién llegados con investigadores sin colaboración. Aquí ya surge, como huella de colaboraciones sucesivas, un lazo de color rojo (de colaboración múltiple) entre los dos nodos titulares de la red, que fortalecen sus vínculos en el centro de la trama. 
Figura 6. Configuración de la red de Team Assembly transcurridas 46 iteracciones de una simulación estándar

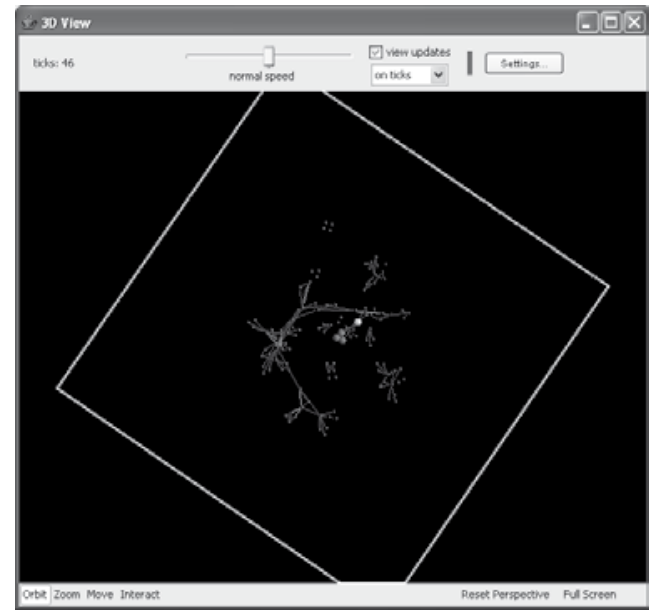

En el transcurso del tiempo, y como vemos en la figura 6, nuevos conglomerados se van sumando y generando dos dinámicas de agrupamiento básicas: por un lado, investigadores que pertenecen a un componente central, y en segunda instancia, otros que quedan aislados en regiones desconectadas de la red mayor.

Figura 7. Graficación acumulativa de conteo de tipos de lazos de la red de Team Assembly

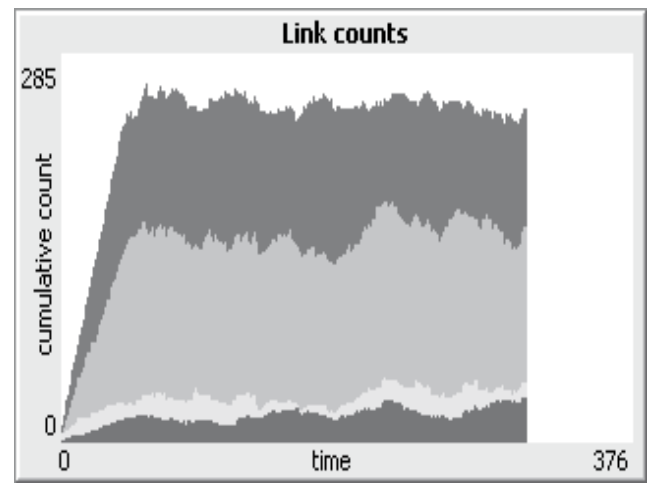

La acumulación relativa de tipos de lazos se puede ver en el monitor de la figura 7. Claramente, los lazos que corresponden a los recién llegados forman el grueso de la den- sidad de la red, seguidos por los de "titular/ recién-llegado", los de "titular-titular", y aquellos que genéricamente refieren a relaciones repetidas en el tiempo.

Por otro lado, como vemos en la figura 8, el porcentaje de los agentes que forman parte del "componente gigante" decrece con el tiempo, lo que indica la creciente fragmentación de la red de acuerdo a los parámetros con que se corrió esta simulación, que son un $40 \%$ para la posibilidad de elegir un titular, y un $65 \%$ para la posibilidad de que si se elige un titular, éste sea un colaborador previo.

Figura 8. Graficación del porcentaje de agentes en el componente gigante de la red de Team Assembly

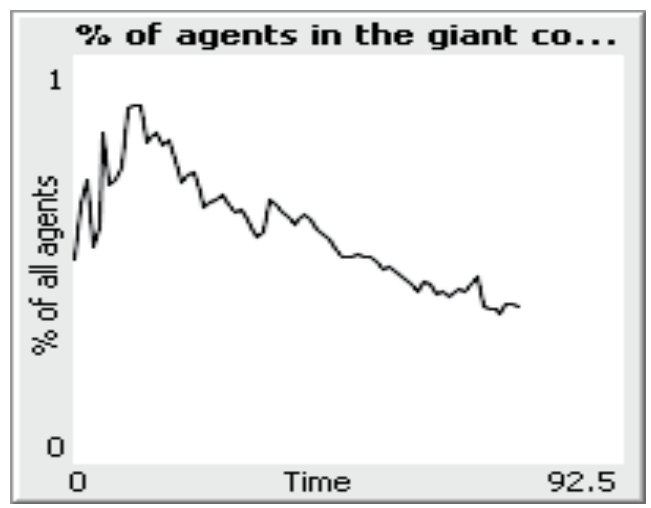

El modelo captura dos características básicas de las redes de colaboración que pueden influir o frenar la innovación en las empresas creativas mediante la variación de los valores de $P$ y $Q$. La primera es la distribución del tipo de conexión entre los colaboradores, que se puede ver en la trama el conteo de tipos de lazos. Un exceso de los vínculos del tipo "recién llegado-recién llegado" (graficado con azul) podría indicar que un campo no se está aprovechando de los miembros con experiencia. Por otra parte, una multitud de colaboraciones de repetición (rojo) 
o de enlaces del modo "recién llegado-titular" (amarillo) pueden reflejar una falta de diversidad en las ideas o experiencias. El segundo atributo es la conectividad global de la red de colaboración. Por ejemplo, de muchos campos académicos se dice que se componen de "colegios invisibles" de comunidades académicas conectadas entre sí. Por el contrario, las redes de patentes tienden a constituirse en sectores aislados, formando cadenas de inventores.

Como muestra la figura 9, el tamaño promedio de los componentes de la red sufre grandes oscilaciones, pero tiende, con discontinuidades, a decrecer y estabilizarse por lo bajo en el tiempo.

Figura 9. Graficación del promedio de tamaño de componentes de la red de Team Assembly

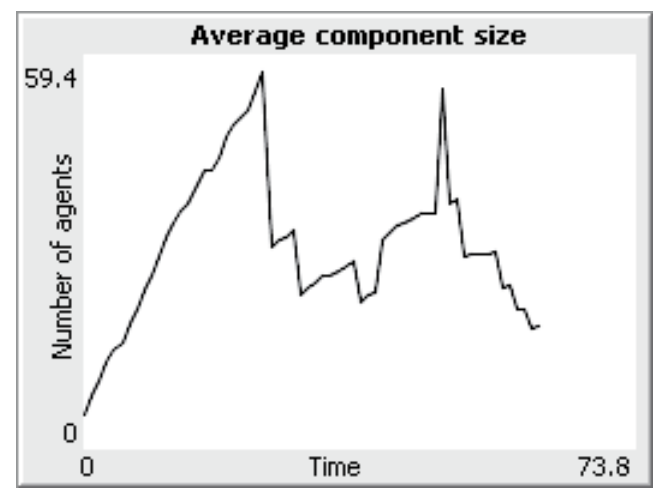

Este tipo de modelos también permite ver las diferentes topologías emergentes en la pantalla. Las nuevas colaboraciones o la "sinergia" entre los equipos tienden naturalmente a ubicarse hacia el centro de ella, y los equipos o grupos de equipos con pocas conexiones a nuevas colaboraciones "flotan" en los bordes de esta estructuramundo. Los recién llegados se ubican, por cuestiones de programación del modelo, siempre en el centro, pero los titulares, que son escogidos al azar, pueden ser ubicados en cualquier parte de la pantalla. Por último, la estructura de redes de colaboración en el modelo puede cambiar drásticamente con el tiempo. Inicialmente, sólo nuevos equipos se generan, ya que en el ámbito de colaboración no ha existido el tiempo suficiente para que los miembros se retiren. Sin embargo, después de un período de tiempo, los agentes inactivos empiezan a retirarse, y el número de agentes se vuelve relativamente estable, los efectos emergentes de $P$ y $Q$ se hacen más evidentes en esta etapa de equilibrio. El final de la etapa de crecimiento está frecuentemente marcado por una caída en la conectividad de la red.

Las preguntas que formula este diseño, con las simplificaciones del caso, son sustanciosas, e implican principios generativos que un análisis tradicional de redes por definición excluye.

¿Es positivo para este intercambio de conocimientos aumentar las exigencias de incorporación de miembros, haciendo que sólo los colaboradores se busquen únicamente entre los más experimentados?

¿Qué tipo de red genera una obsolescencia relativamente exigente, aquí representada por el tiempo de tolerancia para el retiro?

¿Cómo afecta la formación de pequeños conglomerados separados la evolución conjunta de la red y la transmisión de saberes?

La asociación de una configuración reticular con un tipo de proceso generativo arroja, a través de una inducción controlada, pistas que el enfoque clásico de ARS no contempla.

- La primera pregunta, por ejemplo, implica la simulación con un alto porcentaje de titulares exigidos para iniciar 
equipos (86\%). La corrida del modelo arroja resultados contundentes; no hay fragmentación general, y todos los agentes se integran en un gran componente. En cuanto a la distribución de los lazos, predominan aquellos que marcan relaciones múltiples y repetidas en el tiempo entre agente y agente, y prácticamente no hay vínculos con agentes nuevos. En términos más simples, ésta es una red elitista con un alto grado de densidad interna, lo que implica tanto un envejecimiento de la población de expertos como un aumento continuo del promedio del tamaño de los componentes.

- $\quad$ El segundo interrogante se traduce al modelo de manera simple, disminuyendo el período de tiempo impuesto para "expulsar" a un miembro de la red y volviendo a los valores estándar. Lo que sucede es claro; cuando se expulsan agentes con mayor frecuencia, la red se mantiene cohesionada sin más remedio, porque los colaboradores producen o tienen que irse.

- La tercera pregunta, por el contrario, demanda la simulación con un muy bajo porcentaje de titulares exigidos para iniciar equipos $(6 \%)$. Las consecuencias son concluyentes: la red se fragmenta, y muy pocos agentes forman parte del componente central. Esta es una red muy poco elitista, muy propensa a la innovación, pero también muy poco conectada. La alta componentización, y el tamaño muy pequeño de los grupos, hace que la circulación de saberes se vea restringida.

Con este ejemplo, el vínculo entre una configuración de red y un proceso generativo ofrece directivas claras:
- La mayor exigencia de elegir científicos titulares reduce la fragmentación de la red, pero evita la diversificación temática.

- La menor exigencia de elegir científicos titulares aumenta la fragmentación de la red y aumenta la diversificación temática.

- La disminución de la exigencia de producción continua fragmenta prematuramente la red, haciendo que una periferia de científicos poco activos permanezca en el sistema.

Quizás no es tan sencillo traducir estos resultados a conclusiones que tengan una interpretación directa en términos de políticas públicas, pero algunas condiciones para su evolución empiezan a estar claras: si queremos redes con buen flujo de saberes y una edad de investigadores aceptable, ni muy alta ni muy baja, conviene ser moderados en las condiciones temporales para exigir el retiro de los investigadores (no imponer períodos ni muy cortos ni muy largos), pero ser más bien exigentes en cuanto a la incorporación de científicos titulares para los nuevos equipos. Aunque los titulares sean escasos en relación con los recién llegados, mantendrán unidos a los equipos y evitarán la fragmentación de la red.

A pesar de lo prometedor de estas modelizaciones, varios requisitos aparecen pendientes respecto de la evolución futura del ARS y las redes diacrónicas:

- Vincular más amigablemente las interfaces de desarrollo de modelos a los profesionales del análisis de redes, exponiendo "esqueletos" o frames de configuración a los especialistas que quieran dedicarse a profundizarlos. 
- Integrar los dispositivos de análisis a estos modelos, incorporando las métricas básicas (intermediación, grado, cercanía, análisis de cliqués, etc.), a las prestaciones de creación de redes.

- Introducir, a la usanza del modelo SIENA, dispositivos de inferencia estadística sustentados en la evolución de las redes. En la actualidad, no hay paquetes de software que integren la simulación generativa, característica de los modelos basados en agentes, con la simulación inferencial, sustentada en el modelo SIENA.

\section{Algunas conclusiones PROVISIONALES SOBRE LA FACTIBILIDAD DE ESTAS EXTENSIONES}

A pesar del espacio común concedido a este breve repaso, advierto diferentes relaciones, en cada uno de estos apartados específicos, entre la perspectiva epistemológica, la perspectiva analítica y el software demandado:

- En el caso de la extensión hacia lo no aleatorio hay un importante desarrollo de la epistemología y el andamiaje analítico, pero todavía no hay un software que coloque en el centro el análisis distribucional de las redes para extraer conclusiones de sus topologías. Por ahora, diferentes redes no aleatorias pueden generarse con los paquetes estándar, como Pajek, ORA y otros, pero no hay un apa- rato analítico volcado a la catalogación de redes cargadas por los usuarios.

- En el caso de la extensión hacia lo no intuitivo, la perspectiva posible está sólo bosquejada, pero no hay un andamiaje analítico consolidado fuera de la teoría de grafos, y mucho menos un software que pueda soportar globalmente este tipo de cálculos y representaciones.

- En el caso de la extensión hacia la diacronía, tanto la perspectiva como el andamiaje analítico existen en la actualidad, pero el software y las herramientas de trabajo no están integrados en un único paquete, ya que las simulaciones inferenciales (SIENA) están separadas de las plataformas de desarrollo con capacidad simulatoria generativa (NetLogo y otros).

Retomando en parte el concepto bachelardiano de obstáculo epistemológico, podemos decir que nos hemos encontrado, en estos tres casos, con diferentes limitaciones cognitivas que han contribuido a retrasar el desarrollo de las extensiones propuestas. En efecto, tanto la tradición estadística basada en la aleatoriedad relacional, como la concepción naturalista de la idea de red y, en una medida algo menor, la relativa ausencia de un aparato analítico capaz de integrar relacionalmente sucesiones temporales, han sido responsables de este triple confinamiento naturalístico, aleatorista y sincrónico del ARS contemporáneo. 


\section{REFERENCIAS}

Bachelard, G. (1993). La formación del espíritu científico [1948]. México: Siglo XXI Editores.

Bakshy, E. \& Wilensky, U. (2007). NetLogo Team Assembly Model. Recuperado desde [http://ccl.northwestern.edu/netlogo/models/TeamAssembly]. Center for Connected Learning and ComputerBased Modeling, Northwestern University, Evanston, IL.

Barabasi, L. \& Bonabeau, E. (2003). Scale-Free Networks. Scientific American, Vol. 50.

Barabasi, L. (2003). Linked. New York: Plume.

Callon, M., et ál. (1993). Cienciometría. El estudio cuantitativo de la actividad científica: de la bibliometría a la vigilancia tecnológica. Gijón: Trea.

Crane, D. (1972). Invisible Colleges: Diffusion of Knowledge in Scientific Communities. Chicago: University of Chicago Press.

De Federico, A. (2005). El análisis dinámico de redes sociales con SIENA: método, discusión y aplicación. Empiria, Revista de metodología de ciencias sociales, No. 10.

Granovetter, M. (1973). The Strength of Weak Ties. American Journal of Sociology, No. 78.

Guimera, R., et al. (2005). Team Assembly Mechanisms Determine Collaboration Network Structure and Team Performance. Science, Vol. 308, No. 5722, Recuperado desde [http://amaral.northwestern.edu/Publications/Papers/ Guimera-2005-Science-308-697.pdf].

Miceli, J. (2007). Redes libres de escala y su uso en el análisis de datos etnográficos: el caso de la comunidad Tehuelche del Chalía. En: Exploraciones en antropología y complejidad. Buenos Aires: SB.
Miceli, J. (2008). Los problemas de validez en el Análisis de Redes Sociales: algunas reflexiones integradoras. Revista hispana para el análisis de redes sociales, Vol. 14, No. 1. Recuperado desde [http://revista-redes.rediris.es/ html-vol14/vol14_1.htm].

Molina, J.L., et ál. (2002). Redes de publicaciones científicas: un análisis de la estructura de coautorías. Revista hispana para el análisis de redes sociales. Vol. 1, No. 3. Recuperado desde [http://revistaredes.rediris.es].

Padgett, J. (1999). John Padgett application for SFI Research Professor. Recuperado en julio de 2007 desde [http://home.uchicago.edu/ jpadgett/papers/sfi/prop.pdf].

Reynoso, C. (2009). Grafos contra natura: Imágenes y modelos formales versus la prisión del lenguaje (inédito).

Reynoso, C. (2008). Redes libres de escala en ciencias sociales: significado y perspectivas (inédito). Recuperado desde [http://www.analisisredes.com.ar/htm/ Redes\%20libres\%20de\%20escala\%20 en $\% 20$ ciencias $\% 20$ sociales $\% 20 \mathrm{La} \% 20$ Plata.htm].

Roberts, F. (1978). Graph Theory and its Applications to Problems of Society. Filadelfia: SIAM.

Snijders, T. (2001). The Statistical Evaluation of Social Network Dynamics. In: M. E. Sobel \& M. P. Becker (Comp.). Sociological Methodology. Boston London: Basil Blackwell.

Snijders, T. (2005). Models for Longitudinal Network Data. In Carrington, J. Scott, \& S. Wasserman (Comp.). Models and Methods in Social Network Analysis. New York: Cambridge University Press. 
Watts, D. (2004). Six Degrees: the Science of a Connected Age. London: Vintage.

Wilensky, U. (1999). NetLogo. Center for Connected Learning and Computer-Based Modeling, Northwestern University, Evanston, IL. Recuperado desde [http:// ccl.northwestern.edu/netlogo/]. 